\title{
MAPPING OF SEPTAL VENTRICULAR TACHYCARDIA: CLINICAL AND EXPERIMENTAL CORRELATIONS
}

Yuichiro Kawamura, MD

Pierre L. Pagé, MD ${ }^{a, b}$

René Cardinal, $\mathrm{PhD}^{\mathrm{a}, c}$

Pierre Savard, BEng, PhD ${ }^{\mathrm{a}, \mathrm{e}}$

Réginald Nadeau, $\mathrm{MD}^{\mathrm{a}, d}$
In patients with chronic myocardial infarction, ventricular tachycardia originating in the interventricular septum may account for a significant number of arrhythmia recurrences after direct ablative operations. We used total computer-assisted cardiac mapping (epicardial sock, left and right ventricular endocardial balloon electrode arrays) to assess whether tachycardia originating in deep or right-sided layers of the interventricular septum is associated with a specific pattern of epicardial activation sequence. We performed these studies during operations in 18 patients and during experiments in 12 dogs in which a septal myocardial infarction was produced by ligating the anterior septal coronary artery. Intraseptal needle electrodes were plunged into the septum of all animal preparations to generate pace-mapping data and to obtain intraseptal recordings (six preparations) during reentrant ventricular tachycardia induced by programmed stimulation. In addition, pace-mapping data of infarcted canine heart preparations were compared with those of nine healthy heart preparations. In the clinical study, 31 ventricular tachycardias with a septal site of origin were analyzed. Twenty tachycardias displayed an epicardial breakthrough in the area of the interventricular groove, whereas $11 \mathrm{had}$ an epicardial breakthrough in the right ventricular free wall. Biventricular endocardial mapping revealed that left septal endocardial activation preceded right septal activation in the former and that right septal activation occurred earlier in the latter. In the experimental study, 14 ventricular tachycardias (cycle length $146 \pm 34$ msec) were induced by programmed stimulation in 11 infarcted heart preparations. Eight tachycardias displaying an epicardial breakthrough on the right ventricle were found to originate in the right ventricular septal subendocardial layers, whereas six tachycardias in which the epicardial breakthrough occurred on the anterior interventricular groove originated in the left ventricular septal subendocardial layers. The epicardial breakthrough preceded the left ventricular endocardial breakthrough in six tachycardias $(85.7 \%)$ originating in intermediate or right ventricular septal layers, but in only one of five tachycardias originating in the left ventricular septal layers. In the pace-mapping study, the epicardial breakthrough shifted progressively from the right ventricular free wall toward the interventricular groove area in response to pacing from the right, intermediate, and left ventricular thirds of the basal septum. This relationship was similar for infarcted and noninfarcted
From the Centre de Recherche de l'Hôpital du Sacré-Coeur de Montréal, ${ }^{a}$ the Departments of Surgery, ${ }^{b}$ Pharmacology, ${ }^{c}$ and Medicine, ${ }^{\mathrm{d}}$ Université de Montréal, and the Institut de génie biomédical, Ecole Polytechnique de Montréal, ${ }^{c}$ Montreal, Quebec, Canada.

Received for publication July 20, 1995; revisions requested Nov. 25, 1995; revisions received April 30, 1996; accepted for publication May 3, 1996.
Supported by program grant PG-11190 form the Medical Research Council of Canada.

Address for reprints: Pierre L. Pagé, MD, Centre de recherche Hôpital du Sacré-Coeur de Montréal, 5400 Gouin Blvd. West, Montreal, Quebec H4J 1C5, Canada.

J Thorac Cardiovasc Surg 1996;112:914-25

Copyright (C) 1996 by Mosby-Year Book, Inc.

$0022-5223 / 96 \$ 5.00+0 \quad \mathbf{1 2 / 1} / 74709$ 
hearts, although transseptal conduction time was prolonged in infarcted hearts $(45 \pm 10 \mathrm{msec}$ vs $33 \pm 7 \mathrm{msec}, p<0.01)$. Therefore the information integrated from the localization of the epicardial breakthrough and the relative timing between the epicardial and the left ventricular endocardial breakthroughs can be used to estimate the depth of the site of origin of septal ventricular tachycardias. This study confirms that a three-dimensional view of the substratum of ventricular tachycardia can be derived from simultaneous epicardial and left ventricular endocardial mapping and can provide a superior basis for therapeutic interventions. ( $J$ Thorac Cardiovasc Surg 1996;112:914-25)

$T_{\text {te }}^{\text {he }}$ he development of intraoperative mapping techniques has led to successful surgical treatment of ventricular tachycardias (VTs) associated with coronary artery disease and prior myocardial infarction. ${ }^{1-3}$ Sites of VT origin have been most frequently localized in left ventricular (LV) subendocardial layers, ${ }^{1,4,5}$ and procedures aimed at ablating this type of substrate (subendocardial resection, cryosurgery, catheter ablation) have been widely used to treat patients with recurrent sustained monomorphic VT, ${ }^{3,6-8}$ Several experimental and clinical studies, however, have pointed out an important role of intramural or subepicardial layers in initiating and perpetuating reentrant VT. ${ }^{9-12}$ Using biventricular epicardial and LV endocardial balloon electrode mapping in patients with anterior or inferior infarctions (or both), we $\mathrm{e}^{9}$ have found that the site of origin was localized to subepicardial or deep intraseptal layers in up to $30 \%$ of a series of 47 VT episodes. This experience led us to suggest that a three-dimensional view of the substratum of a given VT can be derived from simultaneous epicardial and $\mathrm{LV}$ endocardial mapping and that the information regarding the epicardial-LV endocardial relationship is useful to guide the surgeon toward successful cryosurgical ablation of complex endocardial, intramural, and epicardial substrates. , $^{2}$

The results of our mapping studies have indicated that the localization of the earliest epicardial and endocardial activations were, in many cases, anatomically consistent; in fact, epicardial mapping could predict the site of origin of virtually all free wall VTs. ${ }^{6,13}$ However, the epicardial and LV endocardial relationship is more complex in the case of septal VTs that occurred in $76 \%$ of our patients ${ }^{14}$ and that can be associated with remote epicardial breakthrough sites. ${ }^{6,9}$ In this series, a subgroup of VTs designated as type 5 were associated with a higher risk of surgical failure, presumably because surgical ablation was performed solely on the LV septal surface either by endocardial resection or by cryosurgery. ${ }^{6,15}$ Type 5 VTs were characterized by the occurrence of the epicardial breakthrough in the right ventricular (RV) free wall and the fact that the breakthrough preceded the earliest LV endocardial activation. $^{9}$

The purpose of the current study was to investigate the relative timing of epicardial as well as RV and LV endocardial activations in patients with septal VT through the use of both RV and LV balloon arrays. In addition, these mapping data were compared with those obtained during VT caused by reentry in a canine model of septal infarction. Relative timing relationships were investigated in the canine hearts during pacing from left, right, and intermediate septal sites in the presence of a septal infarct.

\section{Methods}

Clinical study. Eighteen consecutive patients in whom intraoperative computer-assisted activation mapping was performed to guide the surgical therapy of refractory sustained monomorphic VT were selected for this study on the basis of the occurrence of at least one VT displaying an LV endocardial breakthrough on the interventricular septum. All patients, aged $55 \pm 10$ years ( 16 men, two women), had a prior myocardial infarction that was anteroseptal in 12 and inferior in six patients. With the patient supported by normothermic cardiopulmonary bypass with a membrane oxygenator, multiple electrode mapping of ventricular activation was accomplished during VT by means of a previously reported technique that is briefly described herein. ${ }^{9}$

Canine preparations of septal infarction. In 15 dogs anesthetized with sodium thiopental and halothane, the heart was exposed through a left thoracotomy at the fourth intercostal space under sterile technique and the pericardium was incised. The anterior septal coronary artery was identified at its origin near the bifurcation of the left anterior descending and the circumflex coronary arteries and was permanently occluded. The pericardium 


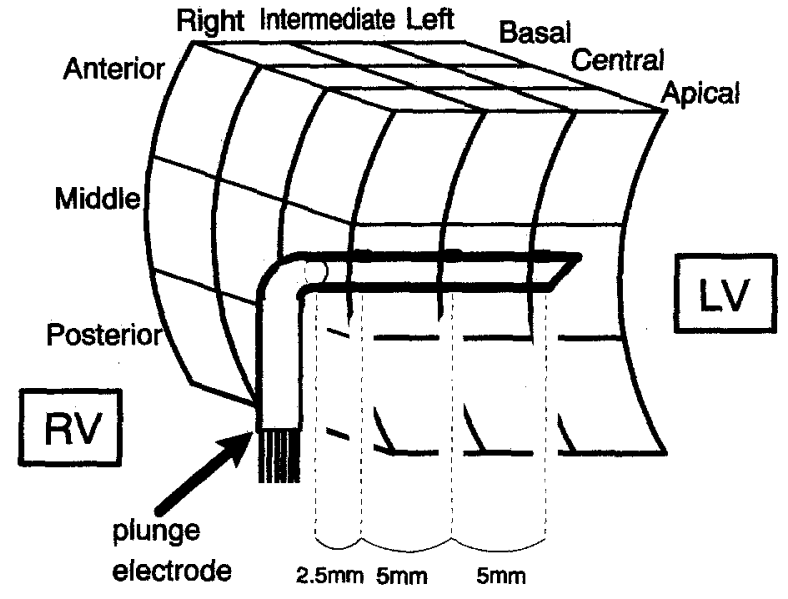

Fig. 1. Schematic representation of the interventricular septum featuring a conceptual division into 27 sites. A rectangular needle electrode containing three bipolar contacts ( $1 \mathrm{~mm}$ interelectrode distance), one at each $5 \mathrm{~mm}$ commencing at $2.5 \mathrm{~mm}$ from the angle, is shown plunged into the apical middle septal block from the RV aspect.

and chest were then closed and postoperative care was given until the day of the mapping studies.

Experimental procedures. Twenty-one mongrel dogs weighing 25 to $40 \mathrm{~kg}$ were anesthetized with sodium thiopental $(15 \mathrm{mg} / \mathrm{kg})$ and alpha-chloralose $(80 \mathrm{mg} / \mathrm{kg})$ and their lungs were mechanically ventilated with a Harvard respirator (Harvard Apparatus Co., Inc., S. Natick; Mass.). Anesthesia was maintained with alpha-chloralose as required. Of the 21 animals, nine were healthy and 12 were studied 3 days after the induction of a septal myocardial infarction, as described earlier (three of the 15 animals died suddenly 24 to 48 hours after occlusion of the anterior septal coronary artery). The heart was exposed through a median sternotomy and suspended in a pericardial cradle. Pairs of Teflon-coated silver wires were sutured onto the RV outflow tract and the LV anterior wall for programmed extrastimulation. Ventricular electrograms, surface leads I, III, and $\mathrm{V}_{6}$ electrocardiograms, and systemic arterial pressure were monitored on a Nihon Kohden polygraph (Nihon Kohden Corp., Tokyo, Japan) and stored on magnetic tape (Hewlett-Packard instrumentation recorder, Hewlett-Packard Co., Andover, Mass.) throughout the experiments. After heparin administration ( $3 \mathrm{mg} / \mathrm{kg}$ ), the aorta and both cavae were cannulated directly, and normothermic cardiopulmonary bypass was instituted with a bubble oxygenator. The epicardial surface of the heart was covered with a sock array of 128 unipolar electrodes. The electrode identification numbers were verified to confirm the anatomic orientation of the sock array over the epicardium. Sixty-four unipolar electrograms were recorded from the LV endocardial surface by means of an inflatable balloon (Institut de génie biomédical, Ecole Polytechnique, Université de Montréal) introduced in the intact $L V$ from the left atrium. 9,16 Then the RV cavity was exposed through a wide incision in the right atrial free wall. Custom-made rectangular needles containing three pairs of bipolar electrodes (distal, intermediate, and proximal plunging position) with 5 $\mathrm{mm}$ interelectrode distance and $2.5 \mathrm{~mm}$ distal-to-proximal pair distance were plunged into nine septal blocks from the RV aspect (Fig. 1) for pacing (12 preparations) and recording (six preparations). The distance between each needle varied from 1.0 to $2.5 \mathrm{~cm}$. Thus this experimental setting allowed for pacing and recording from three different levels for each block (left, intermediate, and right), yielding data for a total of 27 intraseptal sites (Fig. 1). A specially designed 63 -electrode balloon was also introduced into the right ventricle across the tricuspid valve in all preparations.

At the end of the mapping experiment, the animal was put to death and the heart was excised with plunge needles still in place to verify their positions. Data from inadequately positioned needles were deleted from the analysis. In the infarct group, the excised heart was cut into $5 \mathrm{~mm}$ thick slices, which were incubated at $37^{\circ} \mathrm{C}$ for 45 minutes in a buffered 2,3,5-triphenyltetrazolium chloride solution. This allowed the identification of the position of the necrotic mass in relation to the plunge needles. The area of necrosis was measured in each specimen by planimetry and the necrotic mass was calculated as a percent fraction of the total LV myocardial mass.

Pacing protocols. The animals in both groups were subjected to intraseptal pacing trials by stimulation from each bipolar electrode of the plunge needles (cycle length of $300 \mathrm{msec}$ ). Stimuli were rectangular pulses of $2 \mathrm{msec}$ duration and twice diastolic threshold intensity. Programmed cardiac stimulation was performed to induce VT in the infarct preparations. Up to three extrastimuli were applied after a train of 10 beats at cycle lengths of 300 and 250 msec. Each extrastimulus was given at a progressively shorter coupling interval in $5 \mathrm{msec}$ decrements until monomorphic VT was induced.

Mapping procedure. Electrograms were recorded in the unipolar mode simultaneously from the epicardium and the endocardium with reference to Wilson's central terminal. Intraseptal recordings were made in the bipolar mode because needle electrodes were already connected for bipolar pacing purposes. Activation sequences were displayed on polar epicardial and endocardial grids (Fig. 2).

In the clinical setting, epicardial and endocardial recordings were performed in the same manner as described earlier for the animal experiments except that only 63 electrodes were fixed on the human sock electrode. In addition, RV endocardial mapping was performed in seven patients with an RV balloon-similar to the one used in the experimental setting except for size-which was inserted in the RV cavity across the right atrium and the tricuspid valve after bicaval cannulation.

All electrograms were processed and analyzed by means of a digital data acquisition system based on a micro-VAX host computer (Digital Equipment Corp., Maynard, Mass.) and custom-made software, Cardiomap II (Institut de génie biomédical, Ecole Polytechnique de Montréal, Université de Montréal), an upgraded version of the system described earlier. 6,9,17 The signals were amplified by programmable-gain analog amplifiers with a 0.05 to 200 

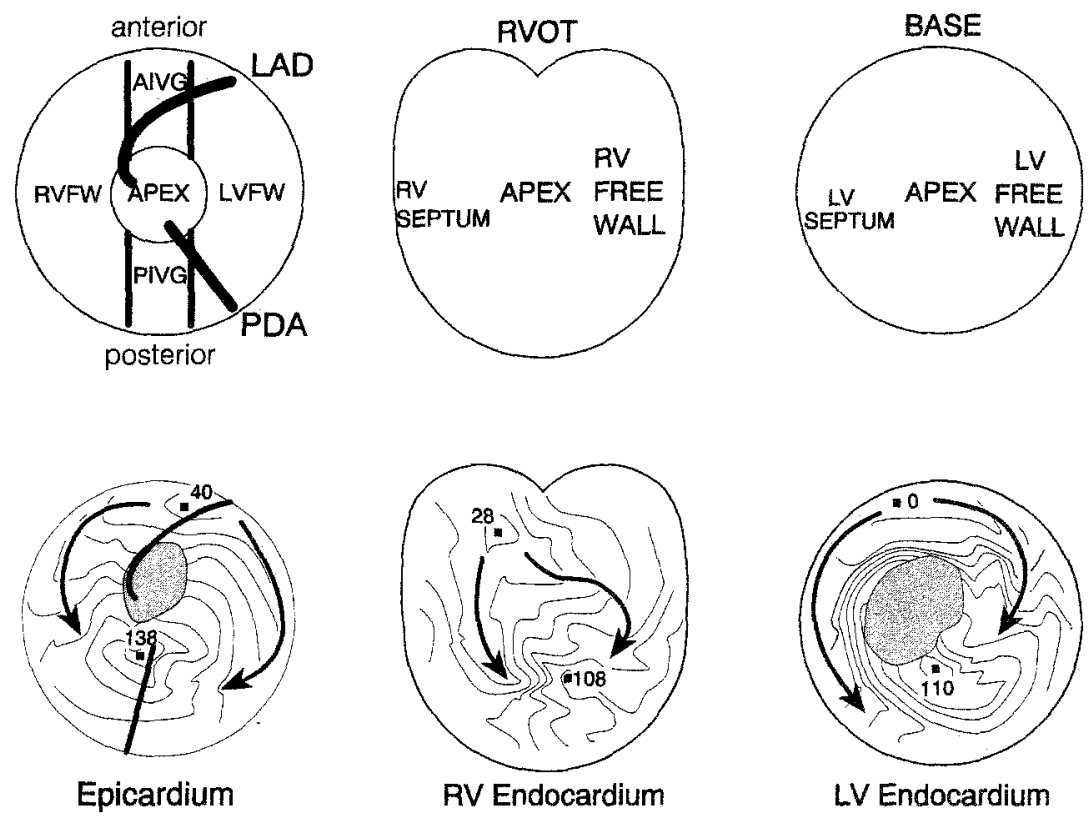

W. infarcted area

Fig. 2. Diagrams showing epicardial and endocardial mapping of VT originating in the left subendocardial layers of the septum in a patient with an anteroseptal infarct (shading). The upper panels show the epicardial, RV endocardial, and LV endocardial mapping grids representing a polar view of the heart in which the base is at the periphery of the circle and the apex is at the center. The epicardial surface is classified into five areas: RV free wall area $(R V F W), L V$ free wall area $(L V F W)$, anterior interventricular groove area $(A D G)$, posterior IVG area $(P I V G)$, and apical area $(A P E X)$. The endocardial grids display their corresponding septal and free wall aspects. Semicircular upward extensions on the RV endocardial grid indicate the balloon prolongation into the RV outflow tract (RVOT). The lower panels show VT activation sequence maps with isochrone lines drawn at $10 \mathrm{msec}$ intervals. Activation sequence is typical of a left septal VT pattern. $L A D$, Left anterior descending coronary artery; $P D A$, posterior descending artery.

$\mathrm{Hz}$ bandwidth, multiplexed, sampled at $1 \mathrm{kHz}$, and converted to a 10-bit digital format. In unipolar electrograms, activation times were determined by a maximum negative slope in excess of $-0.5 \mathrm{mV} / \mathrm{msec}$ as described previously., 18 In bipolar electrograms, activation was taken to occur at the peak of the deflection with the greatest amplitude. ${ }^{4,19}$ Sites at which unipolar or bipolar electrograms did not fulfill these criteria were considered to be unexcitable. Activation times were plotted on their corresponding grids to allow construction of isochronal maps by means of previously described methods. 9,17

Data analysis. The localization of epicardial breakthrough was classified as follows (Fig. 2, left upper panel): the RV free wall area, the LV free wall area, the anterior and posterior interventricular groove (IVG) areas, and the apical area (APEX). The anterior and the posterior IVGs were defined as a band of tissue encompassing one electrode site adjacent to the left anterior or posterior descending coronary arteries on each side. The APEX was defined as the distal third of the epicardium along the basal-apical axis of the heart.
In the experimental pacing study, the two group of preparations (healthy and infarcted hearts) were compared for (1) the localization of epicardial breakthrough sites, (2) the timing of each breakthrough, (3) the relative timing between the epicardial breakthrough and the LV endocardial breakthrough, and (4) the relative timing between the RV and LV endocardial breakthrough sites. The same parameters were analyzed in the VT study. The activation pattern during VT was analyzed according to the intraseptal recording data. In the pace-mapping study, the incidence of maps with each localization of epicardial breakthrough related to a specific septal pacing site was compared by means of the $\chi^{2}$ test. Activation intervals were compared between the two groups of dogs by analysis of variance. Statistics were expressed as mean \pm standard deviation. A $p$ value of less than 0.05 was considered significant.

The institutional committee on human research approved the clinical study protocol. All experimental procedures were carried out in accordance with the guidelines of the Canadian Council for Animal Care. ${ }^{20}$ 


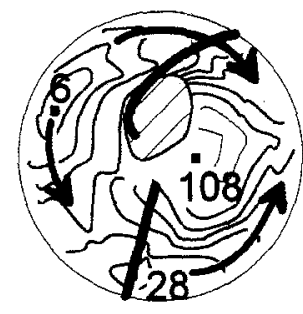

Epicardium

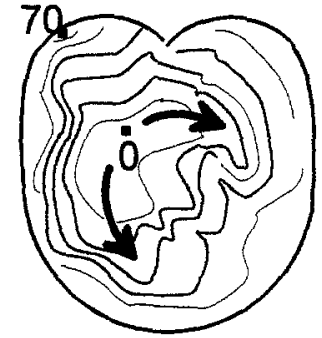

RV Endocardium

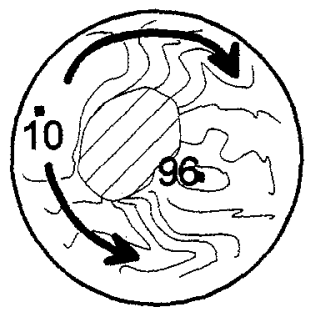

LV Endocardium

Fig. 3. Diagrams showing epicardial and endocardial mapping of VT originating in the right subendocardial layers of the septum in a patient with an anteroseptal infarct. The format is as in Fig. 2.

Table I. Characteristics of VTs

\begin{tabular}{|c|c|c|c|c|c|c|c|c|c|c|}
\hline \multirow[b]{2}{*}{ Dog } & \multirow{2}{*}{$\begin{array}{l}\text { Infarct } \\
(\%)\end{array}$} & \multirow[b]{2}{*}{$V T$} & \multirow{2}{*}{$\begin{array}{c}C L \\
(m s e c)\end{array}$} & \multirow{2}{*}{$\begin{array}{l}\text { Intraseptal } \\
\text { recording }\end{array}$} & \multirow[b]{2}{*}{ Mechanism } & \multirow{2}{*}{$\begin{array}{c}\text { Site of } \\
\text { earliest activation }\end{array}$} & \multicolumn{4}{|c|}{ Localization and timing (msec) of breakthrough } \\
\hline & & & & & & & $E P I$ & Subgroup & $R V$ & $L V$ \\
\hline \multirow[t]{3}{*}{1} & 4.7 & 1 & 96 & - & - & EPI & RVFW (0) & - & - & $\mathrm{BA}(34)$ \\
\hline & & 2 & & - & - & EPI & RVFW (0) & - & - & $\mathrm{CA}(28)$ \\
\hline & & 3 & 114 & - & - & LV & RVFW (8) & - & - & $\mathrm{BM}(0)$ \\
\hline 2 & 2.0 & 4 & 174 & - & - & EPI & RVFW $(0)$ & - & - & $\mathrm{CM}(10)$ \\
\hline 3 & 0.8 & 5 & 108 & - & - & EPI & RVFW (0) & - & - & $\mathrm{BM}(28)$ \\
\hline 4 & 3.5 & 6 & 186 & - & - & EPI & $\operatorname{AIVG}(0)$ & AIVG-1 & $\mathrm{BA}(58)$ & $\mathrm{CA}(30)$ \\
\hline 5 & 1.1 & 7 & 118 & - & - & RV & RVFW (24) & - & $\mathrm{BM}(0)$ & $\mathrm{CM}(26)$ \\
\hline 6 & 6.6 & 8 & 156 & + & Macroreentry & $\mathrm{BAL}$ & AIVG (32) & AIVG-1 & $\mathrm{BA}(20)$ & $\mathrm{CM}+\mathrm{BA}(10)$ \\
\hline $7^{*}$ & 4.8 & 9 & 142 & + & Focal & BAL & AIVG (18) & AIVG-1 & $\mathrm{BA}(26)$ & $\mathrm{BA}(16)$ \\
\hline \multirow[t]{2}{*}{8} & 8.3 & 10 & 134 & + & Macroreentry & CAR & RVFW (18) & - & AM (22) & BA (30) \\
\hline & & 11 & 166 & + & Focal & LV & ArVG (24) & AIVG-2 & $\mathrm{BA}(36)$ & $\mathrm{BA}(0)$ \\
\hline 9 & 1.2 & 12 & 182 & + & Focal & $\mathrm{CMM}$ & RVFW (12) & - & $\mathrm{AM}(8)$ & CM (14) \\
\hline 10 & 3.8 & 13 & 190 & + & Focal & LV & $\operatorname{AIVG}(26)$ & AlVG-2 & $\mathrm{CA}(42)$ & CM $(0)$ \\
\hline $11^{*}$ & 4.8 & 14 & 176 & + & Macroreentry & BAL & AIVG (16) & AIVG-1 & $\mathrm{BA}(16)$ & $\mathrm{CA}(12)$ \\
\hline Mean & 3.8 & & 146 & & & & & & & \\
\hline SD & 2.4 & & 34 & & & & & & & \\
\hline
\end{tabular}

$V T$, Ventricular tachycardia; $C L$, cycle length; $E P I$, epicardial breakthrough; $L V$, left ventricular endocardial breakthrough; $R V$, right ventricular endocardia breakthrough; $B A L$, basal anterior left septal block; $C A R$, central anterior right septal block; $C M M$, central middle intermediate septal block; $R V F W$, right ventricular free wall; $A I V G$, anterior interventricular groove; $B A$, basal anterior area; $B M$, basal middle area; $A M$, apical middle area; $C A$, central anterior area; $C M$, central middle area; $S D$, standard deviation.

*Right bundle branch block pattern seen on normal sinus rhythm map.

\section{Results}

Septal VT: clinical study. In the 18 patients, a total of 31 septal VTs displaying distinct morphologies were mapped by means of epicardial and LV endocardial electrode arrays. Two situations were encountered. In 20 VTs (cycle length $282 \pm 67$ msec), the earliest activation was detected on the LV aspect of the septum, and it preceded the epicardial breakthrough (Fig. 2, lower maps). Conversely, the earliest epicardial breakthrough (localized in the RV free wall) preceded the LV endocardial activation in 11 VTs (cycle length $277 \pm 80$ msec, $p=$ not significant) (Fig. 3).

Fig. 2 shows an example of the first situation in a patient in whom RV endocardial activation was mapped concomitantly with epicardial and LV endocardial activation. LV endocardial activation (0) preceded RV endocardial activation $(28 \mathrm{msec})$ and epicardial breakthrough $(40 \mathrm{msec})$ that occurred in the anterior IVG area. In the 20 VTs of this type, the epicardial breakthrough occurred in either the anterior $(n=8)$ or posterior $(n=10)$ IVG and in the RV free wall in only two VTs. In Fig. 3, illustrating the second situation, the RV endocardial activation $(0)$ preceded both the epicardial breakthrough ( $6 \mathrm{msec})$ and the LV endocardial activation (10 msec). In the 11 VTs of the second type, the epicardial breakthrough usually occurred in the anterior RV free wall $(n=7)$; it was localized in the anterior IVG in only one and in the posterior IVG 


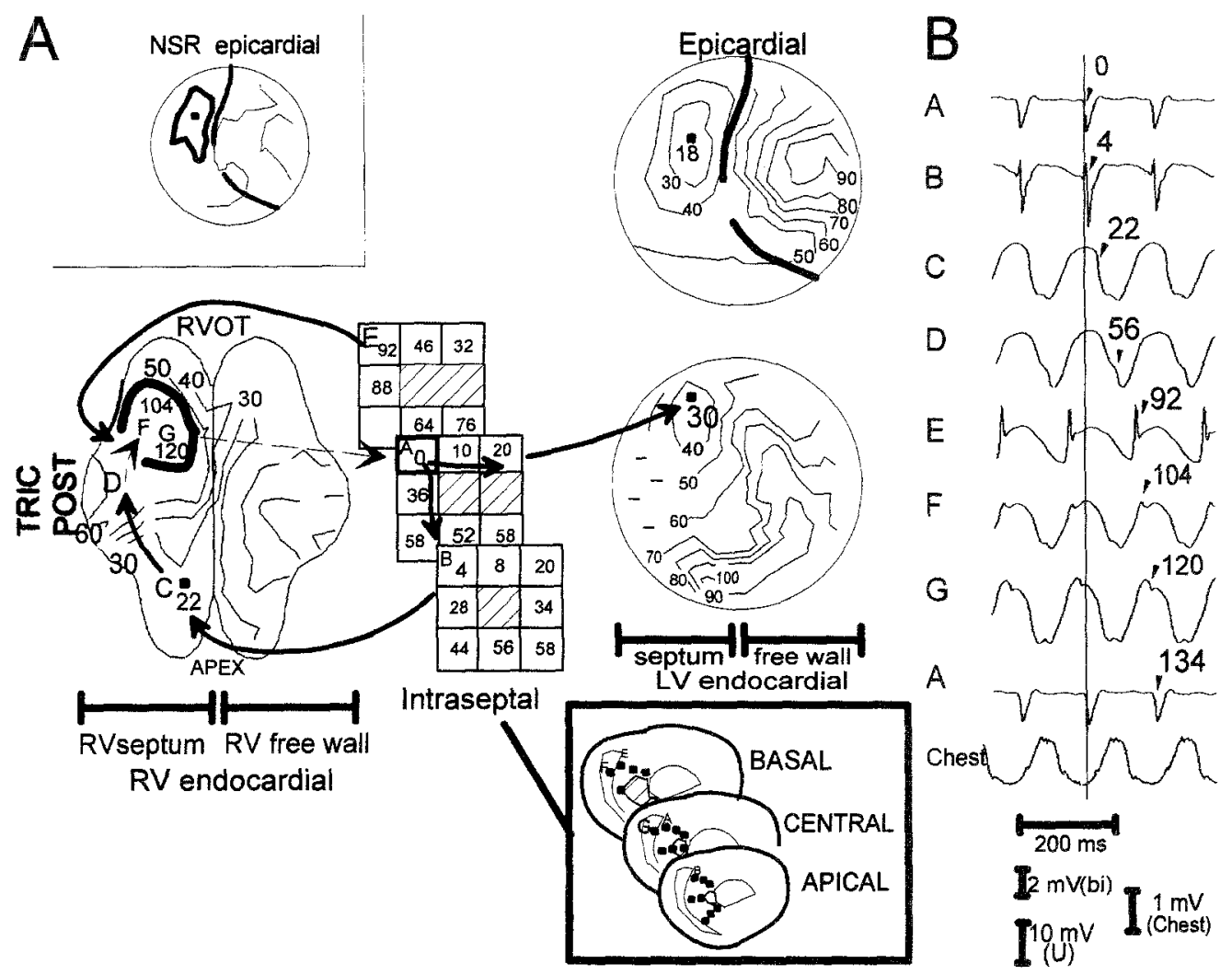

Fig. 4. Epicardial and endocardial maps and selected electrograms of a VT exhibiting an RV free wall epicardial breakthrough (Table I, $\operatorname{dog} 8, \mathrm{VT} 10$ ). A, The left upper inset shows a diagram of the epicardial activation sequence map during normal sinus rhythm (NSR) in dog 8 . The right upper diagram shows the epicardial map during VT 10. The bottom inset indicates the localization of the selected recordings and their relationship to the infarct mass through ventricular cross section. $\mathbf{B}$, Unipolar $(C, D, F$, and $G)$ and bipolar $(A, B$, and $E$ ) electrograms recorded from selected sites on the endocardial surface and intraseptally (see panel $A$ ). Activation times are indicated by arrows. A vertical line was drawn at the zero activation time. bi, Bipolar; $U$, unipolar; RVOT, RV outflow tract.

in three of the remaining VTs $\left(\chi^{2}, p<0.01\right)$. In 13 VTs for which an RV endocardial map was obtained, the right-sided septal activation preceded that of the left-sided septal activation in all (5/5) VTs with an RV free wall epicardial breakthrough, whereas only three of the eight VTs with an epicardial breakthrough in the IVG areas had an earlier RV septal breakthrough. Thus the origin of septal VTs could occur closer to the RV or LV aspect of the septum, yielding in each case a set of distinct mapping characteristics that included the relative timing of the RV endocardial, LV endocardial, and epicardial breakthrough, as well as the localization of the epicardial breakthrough in either the RV free wall or anterior IVG.

Septal VT: animal study. In infarcted heart preparations, the necrotic mass was localized within the basal anterior two thirds of the septum and encompassed $3.8 \% \pm 2.4 \%$ of the total LV mass. Fourteen VT episodes, 13 of which lasted for more than 100 beats, were induced in 11 of the 12 surviving infarct preparations (Table I). Their mean cycle length was $146 \pm 34 \mathrm{msec}$. The epicardial breakthrough was localized in the RV free wall in eight VTs and in the anterior IVG area in the other six VTs. In the latter, the free wall was activated more rapidly and the latest epicardial activation occurred in the posterolateral region of the LV in four VTs, suggesting a right bundle branch block pattern. In the remaining two VTs, propagation occurred in a more balanced fashion between the RV and LV free walls, and the latest activation occurred in the posterior basal RV, thereby suggesting a left bundle branch block pattern. Epicardial breakthroughs localized in the RV 

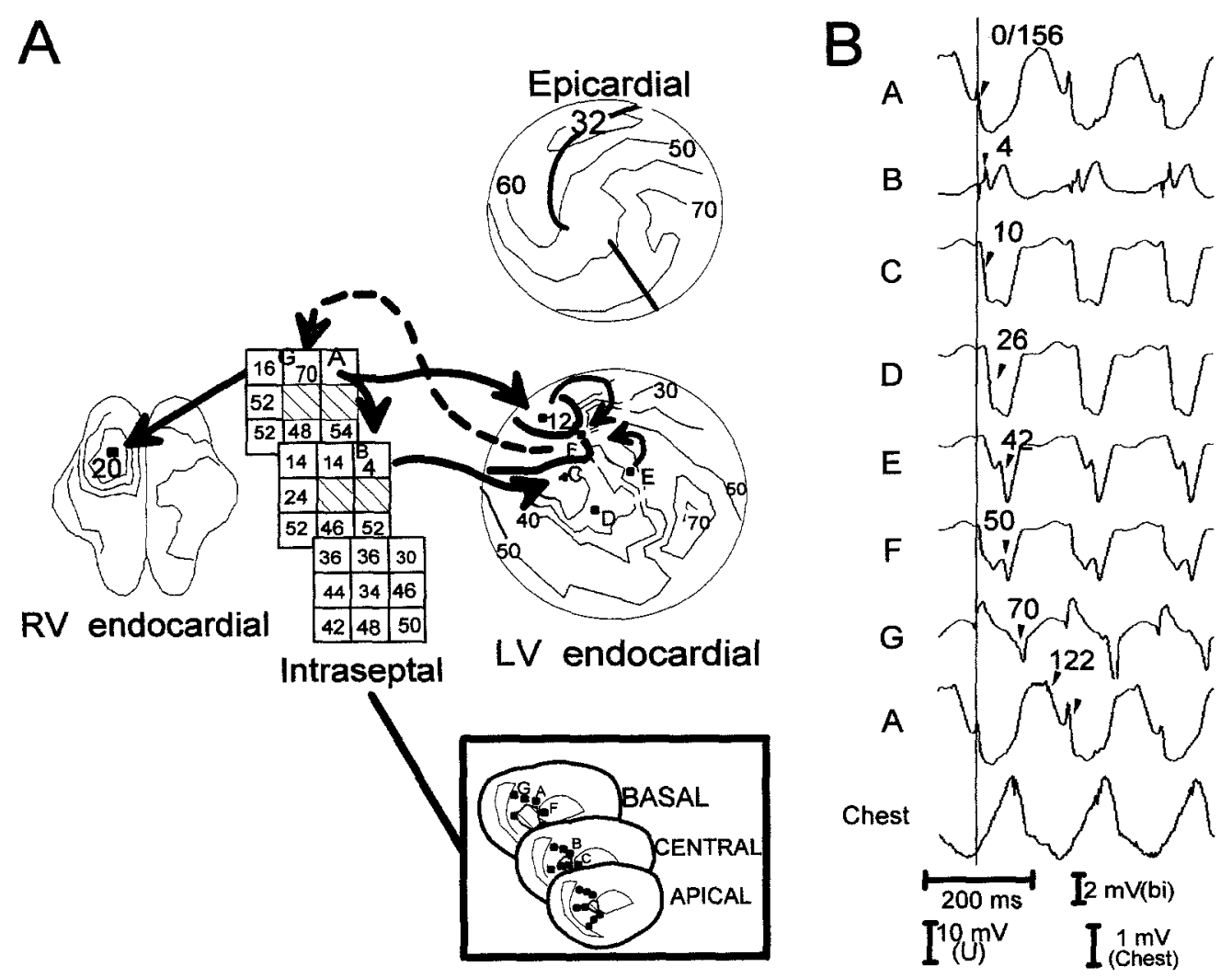

Fig. 5. Epicardial and endocardial maps and selected electrograms of a VT exhibiting an epicardial breakthrough in the anterior IVG area (Table I, dog 6, VT 8). A, The right upper diagram shows the epicardial map during VT. Endocardial maps indicate that the earliest activation (zero time, site $A$ ) occurred within the LV third of the septum. The wave front then divided in two directions to give two separate LV endocardial breakthroughs (dot at $12 \mathrm{msec}$ and site $C$ ). They were followed by two semicircular wave fronts that merged on the basal anterior septum (site $F$ ) and then plunged into the septum (site $G$ ). The RV endocardium was activated passively in a concentric spread from the breakthrough site (20 msec) toward the base of the right ventricle. $\mathbf{B}$, Unipolar $(C, D, E$, and $F)$ and bipolar $(A, B$, and $G)$ electrograms recorded from selected sites on the endocardial surface and intraseptally (see panel $A$ ). Activation times are indicated by arrows. A vertical line was drawn at the zero activation time. The electrogram recorded from site A displayed a double potential (122 and $156 \mathrm{msec}$ ), the second one of which corresponded to the beginning of the next beat. $b i$, Bipolar; $U$, unipolar.

free wall area were associated with the occurrence of the earliest endocardial site of activation in the RV aspect of the septum, whereas epicardial breakthroughs localized in the anterior IVG area were associated with the occurrence of the earliest endocardial site of activation in the LV aspect of the septum.

In six preparations (seven VTs) in which detailed transseptal mapping was performed in addition to epicardial and endocardial mapping, reentry was mapped in three VTs, as illustrated for one displaying an epicardial breakthrough in the RV free wall (Fig. 4) and another displaying a breakthrough in the anterior IVG (Fig. 5). In Fig. 4 the earliest activation $(A, 0$ time) occurred within the septum (RV third). Activation spread out from this septum and was conducted back toward the RV septal surface $(A$ to $D)$, where the maximum delay occurred ( $E, 104 \mathrm{msec} ; F, 120 \mathrm{msec}$ ), leading to generation of the next VT cycle $(A, 134 \mathrm{msec})$. Thus this VT was presumably caused by a reentrant mechanism localized within the RV third of the septum from which a wave front spread out to activate the LV endocardium. The resulting epicardial-endocardial relationship was similar to that seen clinically in patients with type 5 VTs (see Fig. 3 ). Possible involvement of the right bundle branch was suggested by the fact that the epicardial break- 


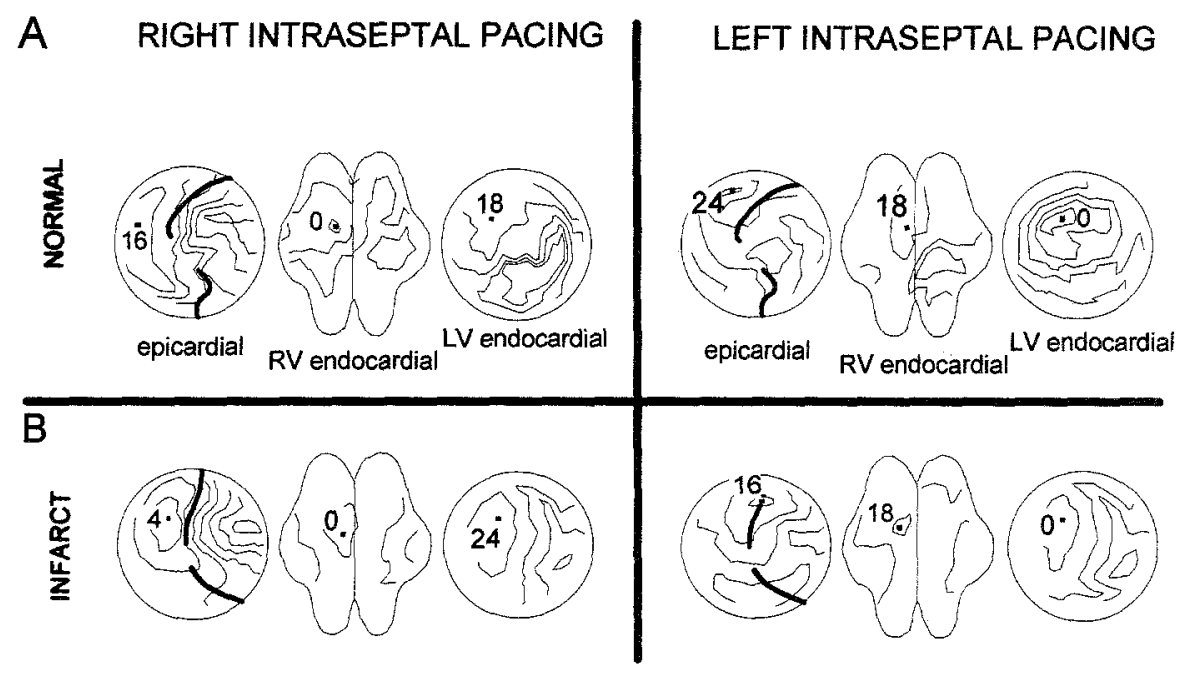

Fig. 6. Maps obtained by pacing the right anterior central and the left anterior central septal sites in healthy and infarcted heart preparations. A, Healthy heart preparation: pacing from the RV (left-hand maps) and the LV (right-hand maps) thirds of the septum. B, Infarcted heart preparation. This infarct preparation was the same as that in which VT 10 was induced (see Fig. 4).

through was localized in the $\mathrm{RV}$ free wall similar to that occurring during sinus rhythm (see Fig. 4, $A$, upper left-hand comer).

In Fig. 5, the earliest site of activation was also localized within the septum, but in its LV third. In contrast to Fig. 4, this VT was presumably caused by a reentrant mechanism located within the LV third of the septum from which a wave front spread out to activate the RV endocardium. The latter was activated in a concentric spread from the breakthrough site $(20 \mathrm{msec})$ and the epicardial breakthrough occurred later $(32 \mathrm{msec})$ in the anterior IVG area. This pattern corresponded to clinical septal VTs originating in left-sided layers of the septum, as illustrated in Fig. 2.

Septal pacing studies. During sinus rhythm, the earliest activation was detected on the LV endocardial aspect of the septum. This activation was followed by activation of the RV septal aspect and by epicardial breakthrough in the RV free wall. This pattern, which is similar to previously reported data, ${ }^{21}$ occurred in all healthy and in most $(10 / 12)$ infarcted heart preparations. In the remaining two infarcted heart preparations, the ventricular activation patterns were altered by bundle branch block. During intraseptal pacing near the RV endocardium in a healthy preparation (Fig. 6, $A$, left), the earliest activation was detected in the corresponding septal region and preceded $\mathrm{LV}$ endocardial activation (by $20 \pm 10 \mathrm{msec}$ ), and the epicardial breakthrough occurred in the RV free wall concomitantly with $\mathrm{LV}$ endocardial activation $(-3 \pm 18 \mathrm{msec})$. The region over which the RV epicardial breakthrough occurred was similar to that in sinus rhythm. During intraseptal pacing near the LV endocardium (Fig. 6, $A$, right), the earliest $\mathrm{LV}$ endocardial activation preceded RV endocardial activation (by $12 \pm 12$ msec) and the epicardial breakthrough occurred in the RV free wall ( $21 \pm 16 \mathrm{msec}$ later) but in a more anterior location that was distinct from the breakthrough area in sinus rhythm. When intraseptal pacing was performed in infarcted heart preparations (Fig. 6, $B$ ), conduction time from the RV to LV endocardial aspects of the septum was significantly increased (from $20 \pm 10 \mathrm{msec}$ in healthy hearts to $28 \pm 9 \mathrm{msec}, p<0.005$ ); the RV epicardial breakthrough preceded the earliest LV endocardial activation (by $6 \pm 16 \mathrm{msec}$ ) (Fig. 6, B, left), as seen earlier with human and animal VTs originating from the RV septal region. This can be appreciated by comparing Fig. 6, $B$, and Fig. 4, which report data during right septal pacing and VT induced in the same preparation and show similar epicardial breakthrough localizations in both. During pacing near the LV endocardial aspect, the relative timings between $\mathrm{LV}$ and RV endocardial activations (10 \pm $12 \mathrm{msec}$ ) and $\mathrm{LV}$ endocardial and RV epicardial breakthroughs ( $18 \pm 13 \mathrm{msec}$ ) were similar to those occurring in healthy hearts. However, the epicardial breakthrough occurred more anteriorly in the RV 
free wall (Fig. 6, B, right) or anterior IVG, a situation that was also encountered in VTs in human beings (see above, Fig. 2) and in animal VTs originating from LV septal segments (see above, Fig. 5).

The presence of an infarct in the interventricular septum decreased the incidence of RV free wall breakthrough during leftward pacing from $58 \%$ to $27 \%(p<0.05)$ and from $97 \%$ to $74 \%(p<0.05)$ during rightward pacing. The incidence of anterior IVG breakthroughs was higher during pacing from leftward sites than from any other site, especially in infarcted heart preparations $(42 \%$ and $10 \%$ for infarcted vs normal preparations, respectively, $p<$ $0.01)$ and was never produced by rightward pacing. The incidence of apical and posterior IVG breakthrough sites was not significantly influenced by the location of the pacing electrode. LV septal pacing from anterior and posterior sites produced epicardial breakthrough sites more frequently localized in the interventricular areas, whereas pacing from left middle segments produced more frequent RV free wall breakthrough sites.

The activation time of epicardial and endocardial breakthrough sites for pacing trials from each septal third indicated that the timing of the RV endocardial breakthrough did not differ between healthy and infarct preparations except during left-sided septal pacing, in which the stimulus to RV endocardial activation interval was significantly greater in the infarct group ( $37 \pm 9$ vs $32 \pm 9 \mathrm{msec}, p<0.001$ ). The stimulus to LV endocardial activation intervals was significantly prolonged in infarcted hearts in response to right, intermediate, or left-sided septal pacing ( $38 \pm 8,28 \pm 9$, and $19 \pm 7 \mathrm{msec}$ vs $48 \pm 9$, $36 \pm 10$, and $28 \pm 10 \mathrm{msec}$, respectively, $p<0.001$ ). Interestingly, the stimulus to epicardial breakthrough intervals did not vary between healthy and infarcted heart preparations or on the basis of the pacing site.

The relative timing of the earliest activations between the epicardium and LV endocardium and the RV-LV endocardial surfaces during VT and during septal pacing shifted toward positive values (i.e., LV endocardial activation preceding) as the pacing sites or the VT site of origin moved leftward. In VTs displaying an epicardial breakthrough in the RV free wall, the RV epicardial breakthrough and RV endocardial breakthrough preceded the LV endocardial breakthrough (by $14 \pm 14$ and $13 \pm 9$ $\mathrm{msec}$, respectively), whereas they followed the LV endocardial breakthrough in VTs with an anterior IVG breakthrough (by $8 \pm 19$ and $22 \pm 14 \mathrm{msec}$, respectively). When pacing was performed from the right side of the septum, the endocardial breakthrough occurred significantly earlier in the infarct group, but the difference was not significant for the other (intermediate and left) septal pacing sites.

\section{Discussion}

The data presented herein support the notion that there is a conceptual continuity between septal VTs in which reentry may (1) occur completely in subendocardial layers on the LV aspect of the septum, (2) involve intraseptal layers, or (3) occur completely in subendocardial layers on the RV aspect of the septum. In the first situation, a complete reentrant circuit can be mapped on the LV endocardial surface; if the circuit is located slightly deeper into the septum, the earliest activation can be detected on the LV endocardial surface. In situations 2 and 3 , activation spreads away from the LV endocardial breakthrough site, which was preceded by the epicardial breakthrough occurring on the RV free wall. The RV epicardial-LV endocardial interval was greater in situation 3 than in situation 2 . This was confirmed by transseptal pacing, as well as by the creation of a septal reentry substrate in canine preparations.

In the present study, we examined epicardial activation patterns during pacing from several intraseptal sites in normal and infarct canine preparations and during VT induced in the infarct preparations. The results showed the following: (1) The incidence of RV free wall epicardial breakthrough sites decreased and anterior IVG area breakthrough sites increased as the pacing site or the VT site of origin moved from the right to the left septum; (2) transseptal impulse propagation was significantly delayed by septal infarction; (3) the location of the epicardial breakthrough was influenced by the existence of a septal infarct; and (4) in the canine septal infarct model, three types of VT could be induced, which displayed epicardial breakthrough sites corresponding to those produced during pacing from the septal region corresponding to the sites of earliest activation during the VT.

The study confirms a previous report by Tweddell and associates ${ }^{22}$ showing that reentrant VT can be induced in the canine anterior septal infarct model. Most canine models of VT are produced by the occlusion of the anterior descending coronary artery, resulting in anterior free wall infarctions. ${ }^{21,23}$ Although excellent to study the reentrant mechanism of ischemic VT caused by a subepicardial 
substrate, the infarct localization does not have any septal extension. ${ }^{23}$ In the human being, the origin of chronic VT is localized to the endocardium and the majority of VTs are associated with septal extension of the infarct. ${ }^{4-7,9,11,14}$ At necropsy of patients with septal VT, Bolick and colleagues ${ }^{24}$ found that the left anterior two thirds or the posterior third of the septum (depending on the occluded artery) were involved by the necrotic process and that the infarct mass per se may act as a barrier against intramural impulse propagation. Moreover, the right half of the septum might be playing a role analogous to that of the subepicardium in free wall VTs. ${ }^{25,26}$ In this regard, $\mathrm{we}^{27}$ have demonstrated that septal infarction profoundly affects $L V$ endocardial activation during sinus rhythm in patients with chronic myocardial infarction. In the $\operatorname{dog}, 75 \%$ of the septal arterial supply is provided by one single artery, the anterior septal coronary artery. ${ }^{28}$ Thus the model of anterior septal artery occlusion appeared to produce VT with an activation pattern closer to the situation seen in human beings. The data reported by Tweddell and coworkers ${ }^{22}$ indicated that among the 15 VTs mapped in their study, the earliest activation occurred on the LV endocardial surface in 11 and on the RV septal surface in three. The activation sequence of the epicardium was characterized by concentric spread of activation, but localization of epicardial breakthrough sites and their relative timing with reference to that of LV and RV endocardial septal breakthroughs were not detailed. Our study shows that the site of epicardial breakthrough was related to the site of earliest septal activation in both the clinical and the experimental situations, whether complete reentrant activity or only a focal pattern could be mapped on the septal endocardial surface. The relationship between RV epicardial and LV endocardial breakthrough sites was determined by the location of the site of earliest septal activation. When activation was first detected at RV septal sites, RV epicardial breakthrough preceded LV endocardial activation. Thus the time relationship between these two breakthrough sites can be used as an indirect indication of the depth of the exit point from the reentrant circuit.

Pace-mapping study. The present study complements that of Smith and associates ${ }^{29}$ who studied epicardial activation patterns in response to pacing from multiple intraseptal sites in the healthy canine heart. They also showed that pacing from rightsided septal segments produced epicardial breakthrough in the right ventricle. In the presence of septal infarction (which was localized in the left half of the septum), the timing of the LV endocardial breakthrough during right septal pacing was significantly delayed in comparison with healthy preparations, whereas timing of the epicardial breakthrough and RV endocardial breakthrough was not affected. In intermediate and left septal pacing trials, the infarct mass disturbed leftward impulse propagation. One possible explanation is that the infarct mass might have acted as a barrier preventing the impulse from capturing the left bundle branch and thereby favoring a breakthrough in the anterior IVG area rather than at the point of insertion of the right bundle branch in the RV free wall. The similarity between the epicardial map during sinus rhythm and that obtained during VT is consistent with retrograde activation of the left bundle branch and antegrade conduction down the right bundle branch. However, preferential conduction related to muscle fiber orientation may also be involved in directing the impulse toward the right paraseptal region (anterior IVG) or free wall. ${ }^{25}$

The present study demonstrates that simultaneous epicardial-LV endocardial mapping during VT associated with infarction in the clinical or experimental setting can predict the localization of the septal site of origin of the VT, obviating the need for RV endocardial mapping: (1) An RV anterior free wall breakthrough preceding the LV endocardial breakthrough predicts a site of origin located in the right subendocardial layers of the interventricular septum; (2) an epicardial breakthrough occurring in the anterior IVG area suggests that the VT originates in the left subendocardial septal layers.

Implications. Because the occurrence of a type 5 VT may influence postoperative results, its preoperative detection appears desirable both for selecting a patient for surgical therapy and for defining the type of ablative procedure to be performed. Body surface potential mapping has been proposed as a method of preoperative identification of VT substrates. ${ }^{30}$ However, it is more sensitive to epicardial than to intramural and intraseptal activation. The present study provides important clues to the interpretation of body surface maps in predicting the sites of origin of VT.

Up until 1989, the technique used in our institution to ablate VT substrates consisted of cryosurgery alone applied regionally to the endocardial half of the LV encompassing the site of earliest activation. ${ }^{6}$ Our concerns regarding the significance of type 5 
$\mathrm{VT}^{15}$ prompted us to modify the surgical technique used for septal VT ablation. This modification consisted of producing transmural freezing injury of the septum by resecting all visible scar from the LV septal surface before the application of cryosurgery inside the margins of this resection. We believe that the cryoprobe should be applied during cold blood cardioplegic arrest to allow a deeper freezing injury and also because myocardial contact of the cryoprobe is facilitated in a flaccid heart. This modification is performed whenever a type 5 VT is identified at mapping. Preliminary information indicates that the success rate of the operation will be improved significantly if these principles are followed..$^{15}$ The present study not only confirms these previous assumptions about type 5 VTs, but also suggests that a three-dimensional view of the substratum of VT can be derived from simultaneous epicardial and LV endocardial mapping, thereby providing a superior basis for therapeutic interventions.

We wish to thank Dr. Robert Cossette for his support of the Arrhythmia Surgery Programme, Mr. Gaetan Tremblay for computer software design and upgrade, Mr. Michel Vermeulen and Mr. Denis Guerette for their technical assistance, and Mrs. Suzan Senechal for her assistance in the preparation of this manuscript.

\section{REFERENCES}

1. Harken AH, Horowitz LN, Josephson ME. Comparison of standard aneurysmectomy and aneurysmectomy with directed endocardial resection for the treatment of recurrent sustained ventricular tachycardia. J Thorac Cardiovasc Surg 1980;80:527-53.

2. Ostermeyer J, Borggrefe M, Breithardt G, Podczek A, Goldmann A, Schoenen JD, et al. Direct operations for the management of life-threatening ischemic ventricular tachycardia. J Thorac Cardiovasc Surg 1987;94:848-65.

3. Cox JL. Patient selection criteria and results of surgery for refractory ischemic ventricular tachycardia. Circulation 1989; 79(Suppl):I163-77.

4. Miller JM, Harken AH, Hargrove WC, Josephson ME. Pattern of endocardial activation during sustained ventricular tachycardia. J Am Coll Cardiol 1985;6:1280-7.

5. Harris L, Downar E, Mickleborough L, et al. Activation sequence of ventricular tachycardia: endocardial and epicardial mapping studies in the human ventricle. J Am Coll Cardiol 1987:10:1040-7.

6. Pagé PL, Cardinal R, Shenasa M, Kaltenbrunner W, Cossette R, Nadeau R. Surgical treatment of ventricular tachycardia: regional cryoablation guided by computerized epicardial and endocardial mapping. Circulation 1989;80(Suppl):I124-34.

7. Selle JG, Svenson RH, Gallagher JJ, Littmann L, Sealy WC, Robicsek F. Surgical treatment of ventricular tachycardia with Nd:YAG laser photocoagulation. PACE 1992;15:1357-61.

8. Breithardt $\mathrm{G}$, Borggrefe $\mathbf{M}$, Wietholt $\mathrm{D}$, et al. Role of ventricular tachycardia surgery and catheter ablation as, complements or alternatives to the implantable cardioverter, defibrillator in the 1990s. PACE 1992;15:681-9.

9. Kaltenbrunner W, Cardinal R, Dubuc M, Shenasa M, Nadeau R, Tremblay G, et al. Epicardial and endocardial mapping of ventricular tachycardia in patients with myocardial infarction. Is the origin of the tachycardia always subendocardially localized? Circulation 1991;84:1058-71.

10. Pogwizd SM, Hoyt RH, Saffitz JE, Corr PB, Cox JL, Cain ME. Reentrant and focal mechanisms underlying ventricular tachycardia in the human heart. Circulation 1992;86:1872-7.

11. de Bakker JMT, van Capelle FJL, Janse MJ, Wilde AAM, Coronel R, Becker AE, et al. Reentry as a cause of ventricular tachycardia in patients with chronic ischemic heart disease: electrophysiologic and anatomic correlation. Circulation 1988;77:589-606.

12. Littmann L, Svenson RH, Gallagher JJ, et al. Functional role of the epicardium in postinfarction ventricular tachycardia: observations derived from computerized epicardial activation mapping, entrainment and epicardial laser photoablation. Circulation 1991;83:1577-91.

13. Pagé $P$, Shenasa M, Cardinal R. Epicardial mapping of ventricular tachycardia to guide regional cryoablation. Circulation 1987;76(Suppl):IV498.

14. Pagé PL, Cardinal R, Dubuc M, Cossette R, Nadeau R. Surgical approach of ventricular tachycardia with deep septal substratum. Circulation 1991;84(Suppl):II195.

15. Pagé PL, Cardinal R, Kaltenbrunner W, Shenasa M, Nadeau R. Epicardial/endocardial patterns associated with failure of surgery for ventricular tachycardia. Circulation 1989;80 (Suppl):II221.

16. Mickelborough L, Harris L, Downar E, Parson I, Gray G. A new intraoperative approach for mapping of ventricular tachycardia. J Thorac Cardiovasc Surg 1988;95:271-80.

17. Bonneau G, Tremblay $G$, Savard $P$, et al. An integrated system for intraoperative cardiac activation mapping. IEEE Trans Biomed Eng 1987;34:415-23.

18. Durrer D, Van Lier AAW, Buller J. Epicardial and intramural excitation in chronic myocardial infarction. Am Heart $\mathbf{J}$ 1964;68:765-76.

19. Claydon FJ, Pilkington TC, Ideker RE. Classification of heart tissue from bipolar and unipolar intramural potentials. IEEE Trans Biomed Eng 1985;BAE-32:513-20.

20. Olfert ED, Cross BM, McWilliams DVM. Guide to the care and use of experimental animals, 2nd ed. Ottawa: Canadian Council on Animal Care, 1993.

21. Pagé PL, Cardinal R, Savard P, Shenasa M. Sinus rhythm mapping in a canine model of ventricular tachycardia. PACE 1988;11:632-44.

22. Tweddell JS, Rokkas CK, Harada A, et al. Anterior septal coronary artery infarction in the canine: a model of ventricular tachycardia with a subendocardial origin, ablation and activation sequence mapping. Circulation 1994;90: 2982-92.

23. Wit AL. Electrophysiological mechanisms of ventricular tachycardia caused by myocardial ischemia and infarction in experimental animals. In: Ventricular tachycardia: mechanism and management. Josephson ME, editor. Mount Kisco (NY): Futura, 1982:33-96.

24. Bolick DR, Hackel DB, Reimer KA, Ideker RE. Quantitative analysis of myocardial infarct structure in patients with ventricular tachycardia. Circulation 1986;74:1266-79. 
25. Streeter DD Jr. Gross morphology and fiber geometry of the heart. In: Berne RM, Sperelakis N, Geiger SR, editors: Handbook of physiology. Section 2: The cardiovascular system, vol 1: The heart. Bethesda: American Physiological Society, 1979:61.

26. Ideker RE, Wagner GS, Ruth WK, et al. Evaluation of a QRS scoring system for estimating myocardial infarct size. II. Correlation with quantitative anatomic findings for anterior infarcts. Am J Cardiol 1982;49:1604-14.

27. Hatala R, Savard P, Tremblay G, et al. Three distinct patterns of ventricular activation in infarcted human hearts-an intraoperative cardiac mapping study during sinus rhythm. Circulation 1995;91:1480-94.

28. Blair E. Anatomy of the ventricular coronary arteries in the dog. Circ Res 1961;9:333-41.

29. Smith WM, Ideker RE, Smith WM, et al. Localization of septal sites in the dog heart by epicardial mapping. J Am Coll Cardiol 1983;1:1423-34.

30. SippensGroenewegen A, Spekhorst $\mathrm{H}$, van Hemel NM, et al. Value of body surface mapping in localizing the site of origin of ventricular tachycardia in patients with previous myocardial infarction. J Am Coll Cardiol 1994;24:1708-24.

Don't miss a single issue of the journal! To ensure prompt service when you change your address, please photocopy and complete the form below.

Please send your change of address notification at least six weeks before your move to ensure continued service. We regret we cannot guarantee replacement of issues missed due to late notification.

\section{JOURNAL TITLE:}

Fill in the title of the journal here.

\section{OLD ADDRESS:}

Affix the address label from a recent issue of the journal here.
NEW ADDRESS:

Clearly print your new address here.

Name

Address

City/State/ZIP
COPY AND MAIL THIS FORM TO: Journal Subscription Services Mosby-Year Book, Inc. 11830 Westline Industrial Dr. St. Louis, MO 63146-3318
OR FAX TO:

314-432-1158

N/ Mosby
OR PHONE:

1-800-453-4351

Outside the U.S., call

314-453-4351 